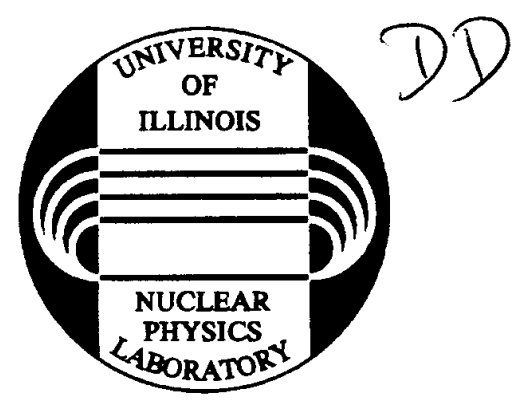

$$
\begin{gathered}
\text { ILL-NPL } 94-002 \\
849408
\end{gathered}
$$

\title{
MEASURED PROPERTIES OF AN OUT-OF-PLANE SPECTROMETER
}

J.B. Mandeville, L.S. Cardman, S.M. Dolfini, W. Kim, R.M. Laszewski, C. N. Papanicolas, S.E. Williamson Nuclear Physics Laboratory, University of Ilinois at Urbana-Champaign Champaign, IL 61820, USA

R. Alarcon, J. Görgen, D. Martinez Arizona State University, Tempe, AZ 85287, USA

K. Dow, M.M. Farkhondeh, D. Tieger, J. Zumbro Bates Linear Accelerator Laboratory, Middleton, MA 01949, USA

\section{Epstein, D. Margaziotis}

California State University at Los Angeles, Los Angeles, CA 90032, USA

A. Bernstein, W. Bertozzi, V. Bhushan, S. Gilad, M. Holtrop, D. Jordon, T. McIlvain, L. Weinstein Massachusetts Institute of Technology, Cambridge, MA 02139, USA

R. Beck, W. Boeglin, E.A.J.M. Offermann University of Mainz, Mainz, Germany

\section{University of Illinois at Urbana Champaign}

\section{Nuclear Physics Laboratory}

\section{Department of Physics}

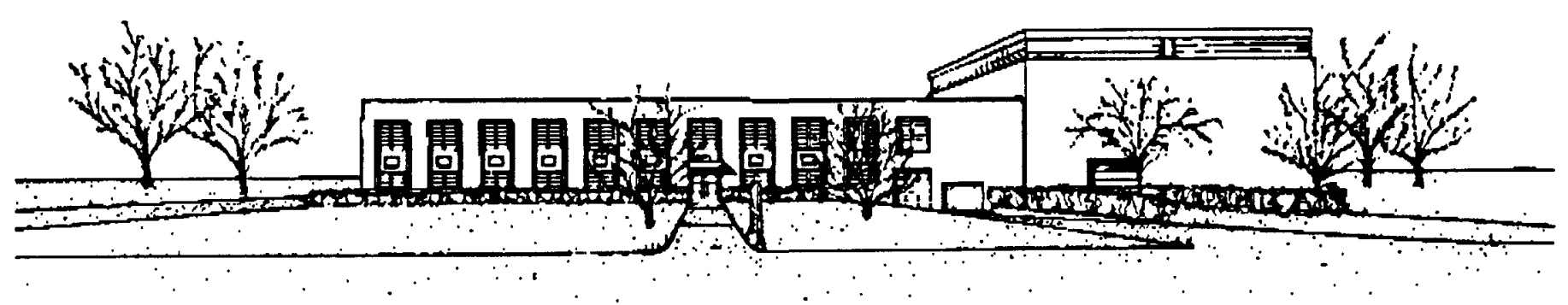




\title{
Measured Properties of an Out-of-Plane Spectrometer
}

\author{
J.B. Mandeville, ${ }^{1}$ L.S. Cardman, ${ }^{2}$ S.M Dolfini, ${ }^{3}$ W. Kim, R.M. Laszewski, \\ C.N. Papanicolas, ${ }^{4}$ S.E. Williamson \\ Nuclear Physics Laboratory, University of Mlinois at Urbana-Champaign, \\ Champaign, IL 61820, USA \\ R. Alarcon, J. Görgen, ${ }^{5}$ D. Martinez \\ Arizona State University, Tempe, AZ 85287, USA \\ K. Dow, M.M. Farkhondeh, D. Tieger, J. Zumbro ${ }^{6}$ \\ Bates Linear Accelerator Laboratory, Middleton, MA 01949, USA \\ M. Epstein, D. Margaziotis \\ California State University at Los Angeles, Los Angeles, CA 90032, USA
}

A. Bernstein, W. Bertozzi, V. Bhushan, S. Gilad, M. Holtrop, D. Jordan;

T. Mcllvain L. Weinstein ${ }^{7}$

Massachusetts Institute of Technology, Cambridge, MA 02139, USA

R. Beck, W. Boeglin, E.A.J.M. Offermann

University of Mainz, Mainz, Germany

\begin{abstract}
We report the results of measurements of the properties of a prototype out-of-plane magnetic spectrometer (OOPS). This spectrometer is one of four identical modules which, together with a support structure, comprise the OOPS cluster. The performance of the spectrometer was found to closely match its design characteristics.
\end{abstract}

PACS numbers: 29.30.Aj, 29.30.Ep

\footnotetext{
${ }^{1}$ Current address; Massachusetts General Hospital, Charlestown, MA 02129, USA.

${ }^{2}$ Current address; Continuous Electron Beam Facility (CEBAF), Newport News, VA 23606 USA

${ }^{3}$ Current address; Arizona State University, Tempe, AZ 85287, USA.

${ }^{4}$ Current address; National and Capodistrian University of Athens, GREECE.

${ }^{5}$ Current address; Emmingen, Germany.

${ }^{6}$ Current address; Los Alamos Meson Physics Facility (LAMPF), Los Alamos, NM 87544, USA.

${ }^{7}$ Current address; Old Dominion University, Norfolk, VA 23529 USA
} 


\section{Introduction}

In a companion paper we have presented the ion-optical and physical design of a magnetic spectrometer that has been specifically tailored for coincident out-of-plane (OOP) particle detection applications [1]. The complete OOPS system will consist of a support structure and four independent spectrometer modules that can be arrayed azimuthally about a symmetry axis located in the scattering plane. The system will be installed in the south experimental hall at the Bates Linear Accelerator Center.

In the present paper we report the measured properties of one of the spectrometer modules as determined during a series of commissioning experiments. An overview of the OOPS module is given in Section 2, and the experimental configuration is discussed in Section 3. The optical performance is evaluated in Section 4, and acceptance and efficiency questions are examined in Section 5.

\section{Overview of the OOPS Module}

The design criteria for the OOPS modules are discussed in detail in the companion report [1]. The maximum momentum is about $800 \mathrm{MeV} / \mathrm{c}$, and the required momentum resolution is modest, being of order one percent. To minimize costs, the design uses existing magnets in a dipole-quadrupole configuration. Each OOPS weighs about 15 tons and is physically compact. The plan view of Figure 1 shows ray trajectories from the target through to the detector package in the dispersive plane as calculated by RAYTRACE $[2,3]$. In this plane the focus is point to point. The focal plane lies at $12.7^{\circ}$ with respect to the central ray. The steep angle of the focal plane with respect to the wire chamber translates into important $2^{\text {nd }}$ and $3^{\text {rd }}$ order terms in the measured detector coordinate expansion of $\delta p / p$.

Figure 2 shows the detector package encased in 6"-thick lead shielding which is supported by 


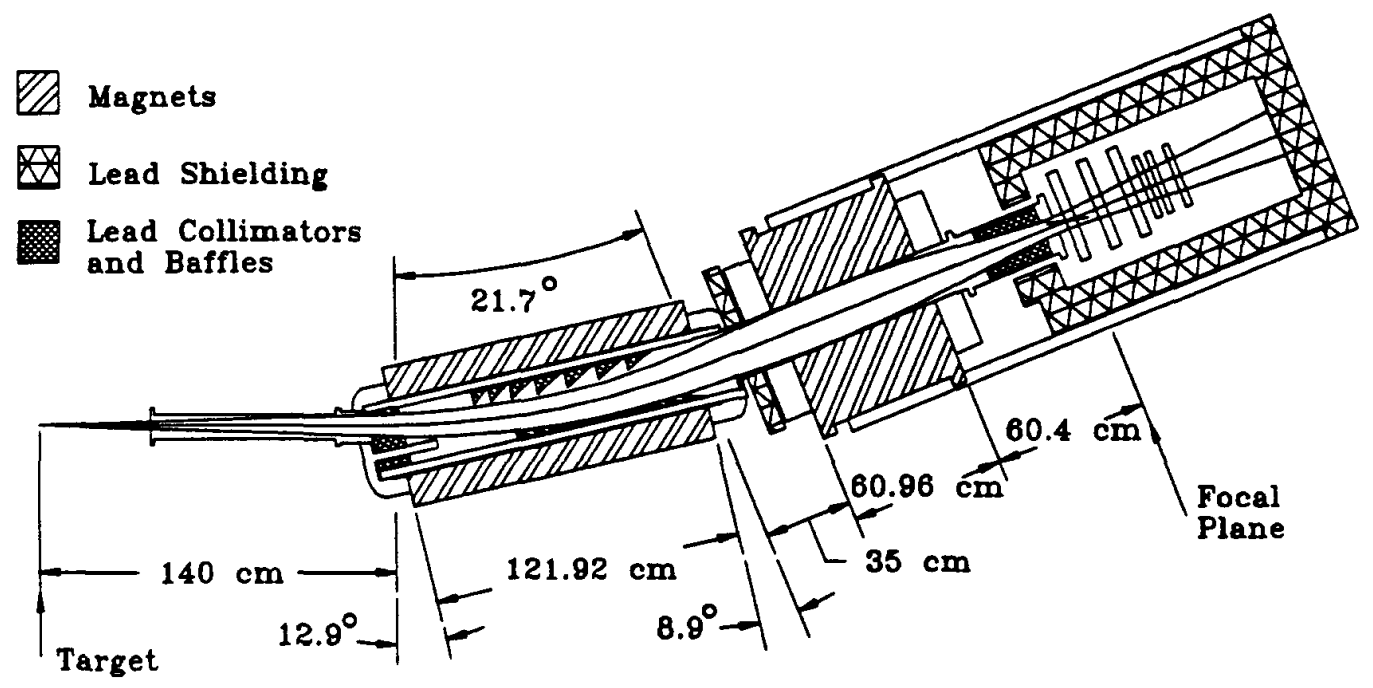

Figure 1: A side plan view of the OOPS traces rays from the target through the spectrometer.

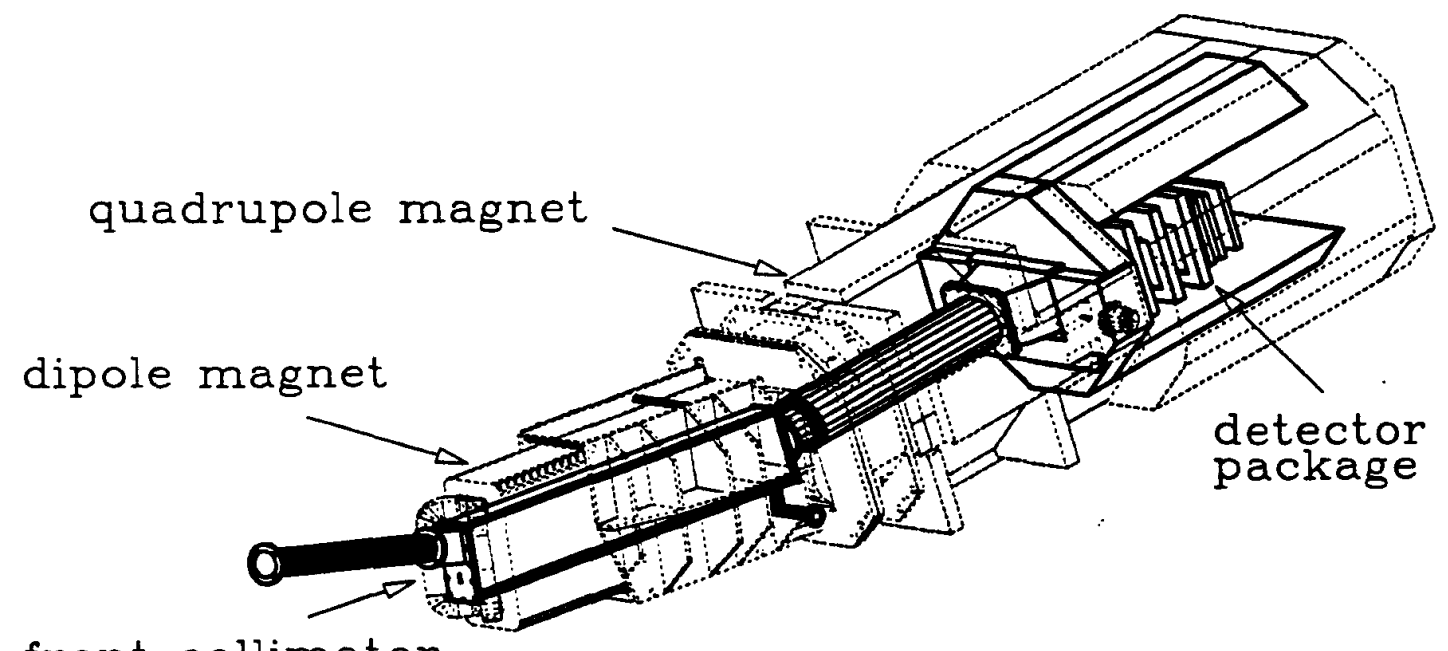

front collimator

Figure 2: The detector package sits inside a 6 " lead house. Solid dark lines show details inside the spectrometer. 
an octagonal steel tube attached to the quadrupole. A $10.25^{n}$-long lead collimator sits inside the last section of the vacuum system. The inside dimensions of this collimator follow the beam envelope defined by the front collimator in the transverse plane and limit the wings of the momentum acceptance in the dispersive plane.

Details of the detector package are shown in Figure 3 [4]. Three horizontal drift chambers

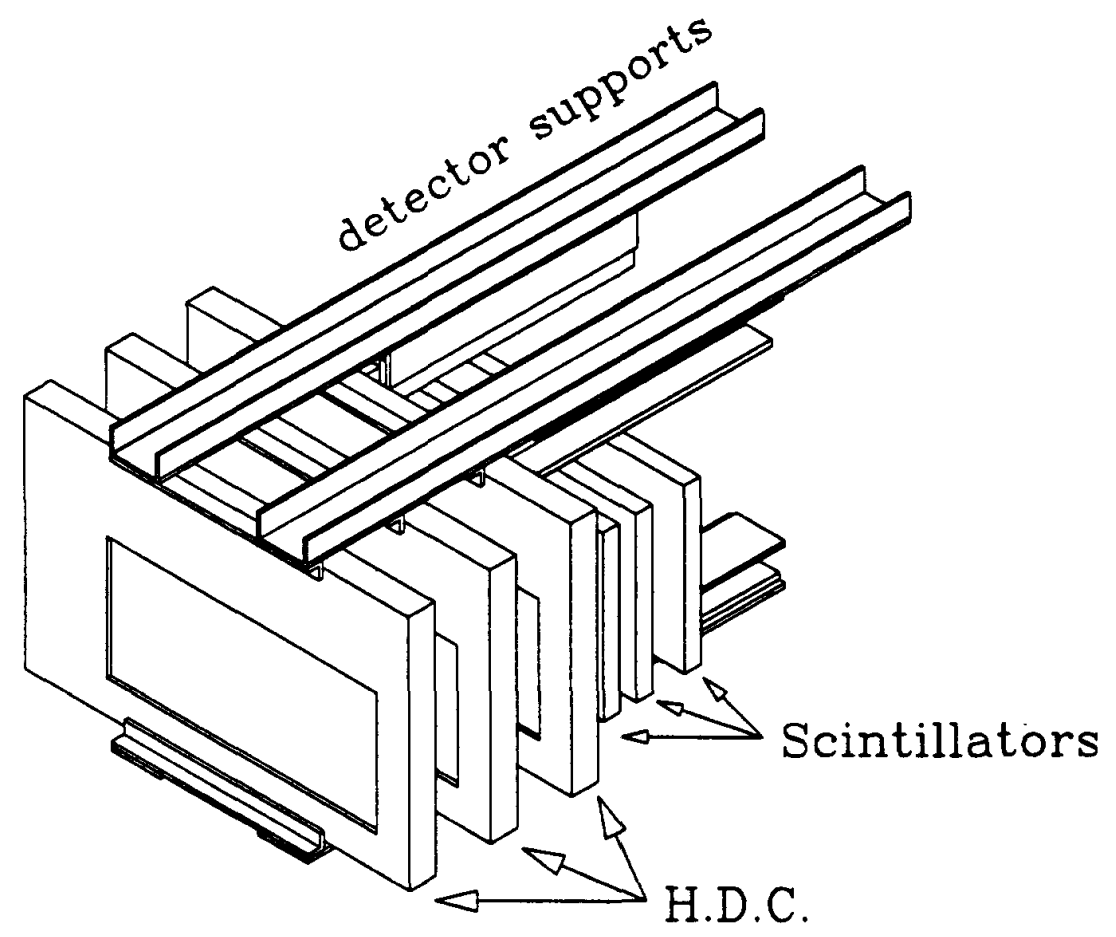

Figure 3: The OOPS detector system [4] consists of three horizontal drift chambers, each containing two transverse wire planes, and three scintillators with photomultiplier tubes on both ends (not shown).

(HDCs) $[5,6]$ are backed by three plastic scintillators. The scintillators have photomultiplier tubes on each end, and the OOPS trigger is formed from a six-fold coincidence among their signals. Each HDC includes two crossed wire planes. By using alternating anode and cathode wires and measuring the cathode wire induced signal amplitudes, each plane locates the intersection point of the particle trajectory in one dimension. Only two sets of crossed wire planes are needed to completely determine a trajectory, but the inclusion of a third chamber boosts the combined HDC efficiency. The detectors are rigidly supported in a 
frame which slides on rails inside the spectrometer. This guarantees reproducible positioning and easy removal and installation.

\section{Experimental Arrangement}

The data discussed in this report were acquired during the summer of 1990 and the spring of 1991 in the North Experimental Hall at the Bates Linear Accelerator Center. During the spring run the electron beam energy was $300 \mathrm{MeV}$ and the OOPS spectrometer was placed at an angle of $48.1^{\circ}$ with respect to the beam exit line. The polarity of the spectrometer magnet could be set for either electrons or protons. Both single arm elastic electron scattering measurements and coincident $\mathrm{H}\left(\mathrm{e}, \mathrm{e}^{\prime} \mathrm{p}\right)$ measurements using the ELSSY spectrometer were performed. The spectrometer alignment was established to better than $1 \mathrm{~mm}$ and $1 \mathrm{mr}$. The accelerator had a $1 \%$ duty factor during the measurement, and energy defining slits limited the beam energy spread to about $0.3 \%$. The beam spot size as viewed on a $\mathrm{BeO}$ target was typically $5 \mathrm{~mm}$. An 80 " thick concrete block was used to provide additional shielding against the beam dump.

The spectrometer shielding was evaluated by recording the luminosity dependence of the counting rates in the detectors. A peak beam current of $0.7 \mathrm{~mA}$ with various thicknesses of ${ }^{12} \mathrm{C}$ targets produced luminosities up to $6 \times 10^{36}\left(\mathrm{~cm}^{2}-\mathrm{s}\right)^{-1}$. Figure 4 shows instantaneous rates in the detectors plotted as a function of luminosity. The trigger rate, formed by a coincidence among three scintillators, scales linearly with luminosity. This rate was essentially zero when the target was removed from the beam, and was negligible even at the highest luminosities if the spectrometer front aperture was blocked. There is very little noise in the trigger from particles outside the angular acceptance of the spectrometer.

All of the delay line singles rates are very similar to the rates plotted for the second $x$ chamber. On the other hand, the slopes and offsets for the singles rates seen in each of the scintillators vary widely. This may be a simple threshold effect. Each scintillator has two 


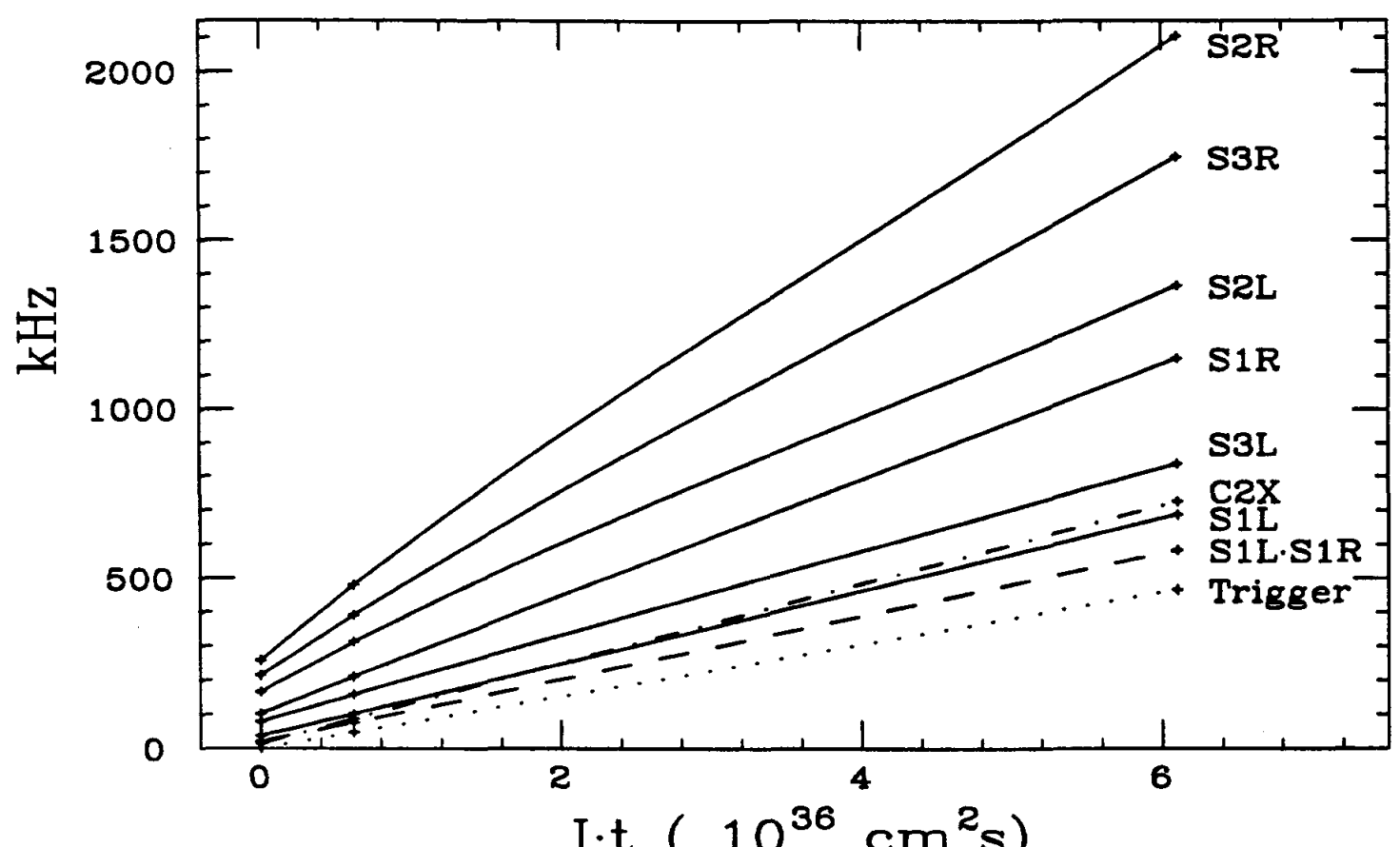

Figure 4: Instantaneous detector rates are plotted versus luminosity for the OOPS in the Bates North Hall with a $250 \mathrm{MeV}$ beam.

phototubes, and their coincidence rate for each scintillator falls in the same range as the delay line singles rates. At the highest luminosity tested, the wire chambers see an instantaneous singles rate of $\approx 300 \mathrm{kHz}$ and the scintillators see singles rates of $100-300 \mathrm{kHz}$.

These measurements allow us to address two important questions concerning the performance of the OOPS module: first, do the singles rates in the wire chambers lead to a significant inefficiency in the detection of trigger events, and second, does the trigger rate produced by the six-fold coincidence of the scintillator phototubes exhibit non-linear behavior with luminosity? Regarding the first question, because there are six wire planes, the efficiency of each plane can be continuously monitored. By requiring signals from just two of three chambers in both the $x$ and $y$ dimensions, the overall wire chamber efficiency was found to be typically greater than $97 \%$. The second question is more difficult to address. The most obvious way that scintillator singles rates can affect the linearity of the trigger rate is to influence it through accidental coincidences. The three-fold accidental coincident rate is 
a simple function of the individual scintillator instantaneous rates and the pulse widths as they enter the coincidence module. We calculate that the accidentals contribute only about $0.1 \%$ to the trigger rate at the highest luminosity tested.

Singles rates can also affect the measured trigger rate in less obvious ways. In fact, a combination of high accidental rates and excessively long pulse widths in our trigger electronics network led to a sizeable (5\%) reduction in the trigger rate during the spring measurements. We have shown, however, that shorter pulse widths at the same rates can achieve trigger network efficiencies of greater than $99 \%$.

\section{Optics}

Transfer matrix element formalism was used to evaluate the target coordinate reconstruction and momentum and angular resolution capabilities of the OOPS module. For reference, the results of the evaluation are summarized in Tables 1 and 2. The measured matrix elements

Table 1: OOPS Target Coordinate Resolutions

\begin{tabular}{|l|l|l|}
\hline Coordinate & Best Resolution & Dominant Resolution Contributions \\
\hline \hline$\delta(=\delta p / p)$ & $0.45 \%$ & $\sqrt{\left(1.3 \delta x_{T}\right)^{2}+\delta \delta_{B}^{2}}$ \\
\hline$\theta_{T}$ & $1.3 \mathrm{mr}$ & $\sqrt{\left(5 \delta x_{T}\right)^{2}+\left(.75 \delta \gamma_{\mathrm{o}}\right)^{2}}$ \\
\hline$\phi_{T}$ & $1.2 \mathrm{mr}$ & $\sqrt{\left(2.5 \delta y_{T}\right)^{2}+\left(.95 \delta \gamma_{s}\right)^{2}}$ \\
\hline
\end{tabular}

and resolutions were found to agree very well with the optical design of the spectrometer [1].

Before proceeding to discuss the results of the optics tests, we briefly review OOPS optics in standard first order matrix formalism. We then discuss the generalization to higher order and our method for determining the matrix elements. Finally, we discuss the results for each target coordinate separately and compare the measured properties with the first order optical design. 
Table 2: OOPS Matrix Elements ${ }^{a}$

\begin{tabular}{|l|l||l|l|}
\hline \multicolumn{2}{|c|}{$\delta(=\delta p / p)$} & \multicolumn{2}{c|}{ Target Angles } \\
\hline MEASURED & TRANSPORT & MEASURED & TRANSPORT \\
\hline$\Delta_{1000}=+4.65 \mathrm{E} 00$ & $\Delta_{1000}=+4.52 \mathrm{E} 00$ & $\Theta_{0100}=-2.907 \mathrm{E}-1$ & $\Theta_{0100}=-2.869 \mathrm{E}-1$ \\
$\Delta_{1100}=+2.17 \mathrm{E}-2$ & & $\Theta_{1000}=-4.05 \mathrm{E} 00$ & $\Theta_{1000}=-3.74 \mathrm{E} 00$ \\
$\Delta_{1200}=+8.2 \mathrm{E}-5$ & & $\Theta_{1100}=-3.0 \mathrm{E}-2$ & \\
$\Delta_{0100}=+4.9 \mathrm{E}-3$ & $\Delta_{0100}=0.0 \mathrm{E} 00$ & & \\
$\Delta_{0120}=-5.4 \mathrm{E}-5$ & & & \\
$\Delta_{0000}=+1.8 \mathrm{E}-1$ & & $\Phi_{0010}=+1.096$ & $\Phi_{0010}=1.103^{b}$ \\
$\Delta_{0020}=+2.1 \mathrm{E}-3$ & & $\Phi_{0001}=0.00$ & $\Phi_{0001}=0.00$ \\
\hline
\end{tabular}

a The notation is explained in Equation 4.

${ }^{6}$ The design matrix elements listed for $\phi_{T}$ represent the point target solution of Equation 3 .

We adhere to the standard conventions used in magnetic optics codes like TRANSPORT. The first order forward transfer matrix for the OOPS is shown below. $T$ indicates a vector at the target, and $\mathrm{F}$ indicates a vector measured in the focal plane region. The $\left\langle x_{F} \mid \theta_{T}\right\rangle$ design value is zero due to the point to point imaging in the dispersive plane. The other matrix elements which are equal to zero vanish as a consequence of midplane symmetry.

OOPS Design Forward Matrix

$$
\left[\begin{array}{l}
x \\
\theta \\
y \\
\phi \\
\delta
\end{array}\right]_{F}=\left[\begin{array}{ccccc}
-0.2869 & 0 & 0 & 0 & +0.2210 \\
-13.74 & -3.485 & 0 & 0 & -2.880 \\
0 & 0 & +2.289 & +0.9068 & 0 \\
0 & 0 & +16.21 & +6.857 & 0 \\
0 & 0 & 0 & 0 & 1
\end{array}\right]\left[\begin{array}{l}
x \\
\theta \\
y \\
\phi \\
\delta
\end{array}\right]_{T}
$$

The inverse matrix cannot be used directly to reconstruct target variables from measured ones because the full five dimensional vector is not detected by the focal plane instrumentation. Only positions and angles are measured. Thus, only two of the three dispersive coordinates $\left(\delta\right.$ and $\left.\theta_{T}\right)$ can be reconstructed. The third dispersive target coordinate must in principle be fixed to allow a solution for the problem. The logical target variable to constrain is $x_{T}$. 
The matrix representation appropriate to the reconstruction procedure is shown below.

\section{OOPS Design Reverse Matrix}

$$
\left[\begin{array}{l}
\delta \\
\theta \\
y \\
\phi \\
x
\end{array}\right]_{T}=\left[\begin{array}{ccccc}
4.524 & 0 & 0 & 0 & 1.298 \\
-3.738 & -0.2869 & 0 & 0 & -5.015 \\
0 & 0 & 6.857 & -0.9067 & 0 \\
0 & 0 & -16.21 & +2.289 & 0 \\
0 & 0 & 0 & 0 & 1
\end{array}\right]\left[\begin{array}{l}
x_{F} \\
\theta_{F} \\
y_{F} \\
\phi_{F} \\
x_{T}
\end{array}\right]
$$

We will show that OOPS has very poor resolution in the non-dispersive plane target interaction position, $y_{T}$. For this spectrometer, we introduce one more matrix, the point target reverse matrix. This corresponds to constraining both the $x_{T}$ and $y_{T}$ target variables. An error analysis, discussed below, shows that this matrix is favored over the one above as a solution for $\phi_{T}$ except in the case of very long targets.

\section{OOPS Point Target Reverse Matrix}

$$
\left[\begin{array}{l}
\delta \\
\theta \\
\phi \\
y \\
x
\end{array}\right]_{T}=\left[\begin{array}{ccccc}
4.524 & 0 & 0 & 0 & 1.298 \\
-3.738 & -0.2869 & 0 & 0 & -5.015 \\
0 & 0 & 1.103 & -2.524 & 0 \\
0 & 0 & 0 & 0 & 1 \\
0 & 0 & 0 & 0 & 1
\end{array}\right]\left[\begin{array}{l}
x_{F} \\
\theta_{F} \\
y_{F} \\
y_{T} \\
x_{T}
\end{array}\right]
$$

These first order matrices illustrate the starting point of the reconstruction procedure and provide a basis for comparison of the measured optical properties with the design values.

We now consider the extension of the first order matrix algebra to higher orders. The subscript $F$ indicates a vector measured in the focal plane region but not necessarily along the focal surface. The expansion makes no assumptions about the location or geometry of the focal surface.

$$
q_{T}=\sum_{i j k l} Q_{i j k l} x_{F}^{i} \theta_{F}^{j} y_{F}^{k} \phi_{F}^{l}
$$


$q_{T}$ is a generalized target coordinate representing one of $[\delta \theta y \phi]_{T}$, as in the first order expansion of Equation 2. The $Q_{i j k l}$ are the coefficients of the expansion for each $q_{T}$ in terms of the measured coordinates $[x \theta y \phi]_{F}$. We will hereafter refer to matrix elements using the convention that $\Delta_{i j k l}$ are the coefficients in an expansion for $\delta, \theta_{i j k l}$ are those for $\theta_{T}$, $\Phi_{i j k l}$ for $\phi_{T}$, and $Y_{i j k l}$ for $y_{T}$. The first order set of these coefficients should closely match the elements of Equation 3.

The expansion in Equation 4 is restricted in two ways:

i) Because of midplane symmetry, the sum $k+l$ must be even when reconstructing the dispersive variables, $\delta$ or $\theta_{T}$, and odd when reconstructing $y_{T}$ or $\phi_{T}$.

ii) For the OOPS studies presented here, the sum $i+j+k+l$ is restricted to be $\leq 3$ for $\delta$ and $\theta_{T}$ and $\leq 2$ for $y_{T}$ and $\phi_{T}$. We have tested higher orders and found their contributions to be negligible.

In principle, the midplane symmetry condition can be violated either by internal misalignments of the magnetic elements or by an overall misalignment of the spectrometer. The OOPS magnetic elements are very well aligned, and the overall alignment of the spectrometer in the North Hall during the test runs was also very good.

To obtain the best set of coefficients $\left(Q_{i j k l}\right)$ for each coordinate, one must generate a set of events with known target coordinates $\left(q_{T}\right)$ which span the full range for which the spectrometer has a high detection efficiency. The coefficients can then be calculated by fitting the focal plane information event by event to the known target variables. For $\delta$, an elastic peak swept across the focal plane at many spectrometer field settings provides the requisite data set. In order to determine coefficients which allow a reconstruction of the angles $\theta_{T}$ and. $\phi_{T}$, the solid angle of the spectrometer was subdivided by installing a 'sieve collimator' [7] in the front aperture which only allows pencil beams to enter the spectrometer at precisely defined angles $[\theta \phi]_{T}$. Our sieve collimator was a 0.5 " steel plate with the holes machined 
in a regular pattern at separations large enough to allow each hole to be resolved in a two dimensional histogram of angles at the focal plane.

The target variables $y_{T}$ and $\delta$ can be reconstructed without such a collimator. However, a separate sieve slit measurement may still be useful to locate the central ray. If the variation in momentum across the horizontal acceptance due to variations in the recoil momentum of the residual nucleus (kinematic broadening) is greater than the spectrometer resolution, the angle $\phi$ should be reconstructed to correct $\delta$ event by event. Kinematic broadening was not a significant consideration for the OOPS data described here, and in general it can be minimized by the choice of a heavy target.

The procedure used in this study is to select an overall order for the expansion and then to systematically eliminate the least important matrix elements one by one, as determined by a $\chi^{2}$ criteria, until the $\chi^{2}$ per degree of freedom begins to rise significantly. If no matrix elements of the highest order included in the original expansion remain, then we have found the smallest set of important coefficients. If any matrix elements of the highest order remain at this point, then we repeat the procedure and allow for the next higher order. We found that this course would lead to the same set of matrix elements regardless of whether the starting set began with order $n$ or $n+1$, where $n$ is the highest order contained in the final results.

There are a number of reasons why it is desirable to rid the expansion of unnecessary coefficients. First, an over-parameterized fit may in fact work less well than a reduced set. This is especially true when one determines matrix elements from a discrete set of known target coordinates and then applies the matrix to data which contains a continuous set of target variables. Within a TURTLE[8] simulation, the reduced set of matrix elements which contribute significantly to the improvement of a fit works as well, and sometimes better, then the full original set. Second, we want to reduce our susceptibility to errors introduced by the uncertainty in the focal plane coordinate offsets which orient the coordinates to the 
central ray. Lastly, the inclusion of uncertainties like multiple scattering and measurement errors causes many coefficients to have errors of $100 \%$ or more, and these are eliminated in our fitting procedure.

As mentioned briefly above, the first OOPS experiments on the nucleon and on deuterium impose only modest momentum resolution requirements. These experiments, together with physical constraints and cost considerations, established a momentum resolution goal of order one percent. Our optical studies indicate that this design goal has been met.

To measure the OOPS intrinsic momentum resolution, we used a carbon filament ${ }^{8}$ as a target (oriented horizontally in the non-dispersive direction $y_{T}$ ). The size of the interaction region on the target in the dispersive plane is generally the largest single contribution to the momentum resolution uncertainty. By using a carbon filament target, we limited the size of the interaction region to one millimeter.

To determine the matrix elements for $\delta$, we swept the carbon elastic electron scattering peak across the focal plane by incrementally varying the spectrometer field to cover a $20 \%$ bite in $\delta$. While the resolution on a carbon filament ( $1 \mathrm{~mm}$ target interaction size) is superior to that on a carbon foil ( $5 \mathrm{~mm}$ interaction size), we found that matrix elements determined from elastic sweeps on the two targets worked equally well. Also, matrix elements determined from a $10 \%$ central segment of the momentum bite extrapolated well to the outer portions of the bite, so elements derived from the full bite undoubtably provide a reasonably good reconstruction of resolution far out onto the wings of the OOPS momentum acceptance.

Figure 5 shows the $\chi^{2}$ per degree of freedom versus the number of coefficients in the expansion for the fit to the carbon foil data. We chose to include seven coefficients in our parameterization of $\delta$. Fourth order coefficients were tested and found not to contribute. The matrix elements for $\delta$ which are quoted in this paper are derived from the elastic sweep

\footnotetext{
${ }^{8} \mathrm{~A}$ pencil lead was used for the target. Chemical analysis reveals that the pencil lead is $43.81 \%$ carbon and $2.22 \%$ hydrogen by weight. The rest is unknown. Future measurements of this kind should use a more isotopically pure substance.
} 


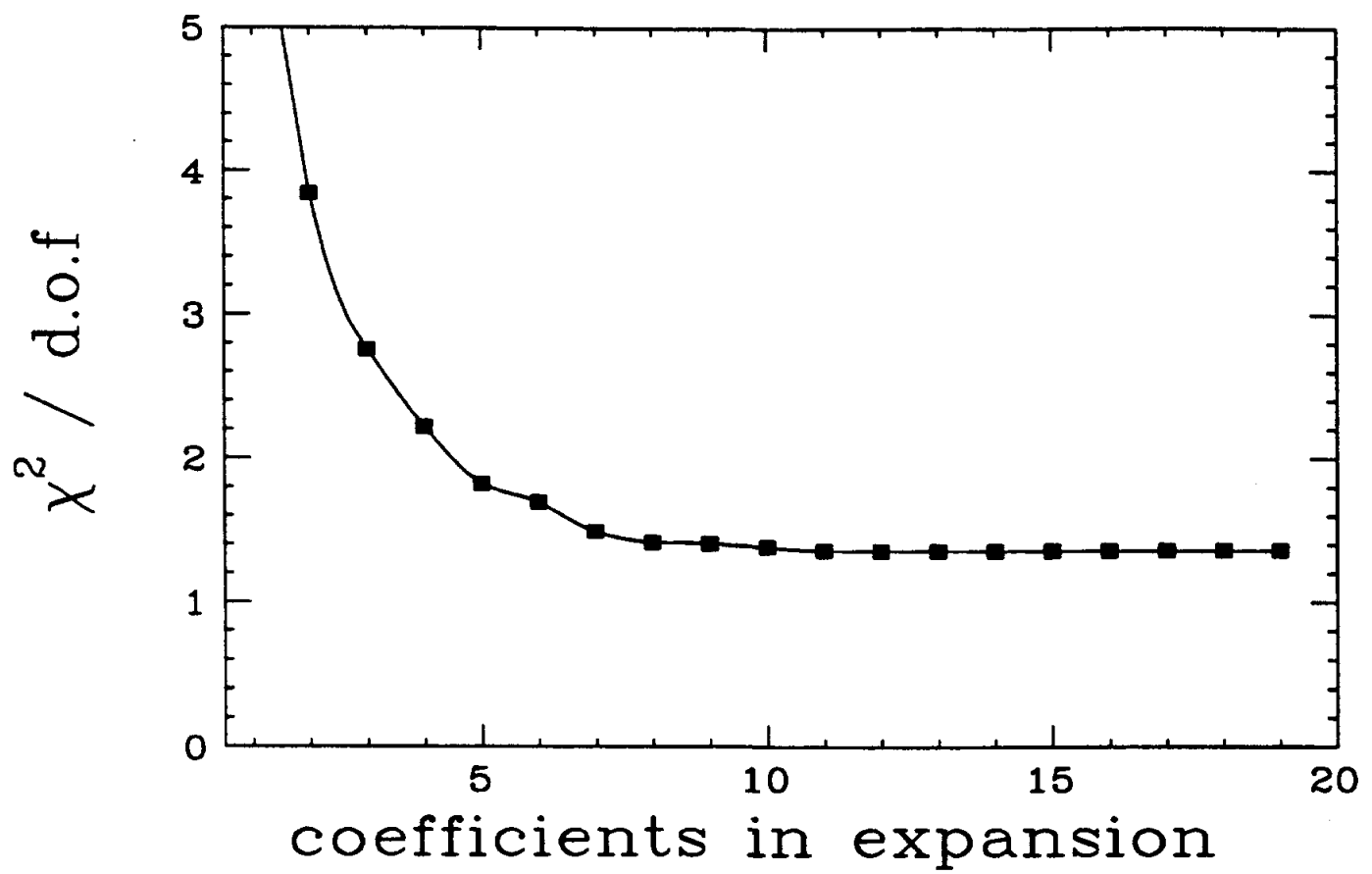

Figure 5: The $\chi^{2}$ per degree of freedom is plotted versus the number of coefficients for a third order expansion of momentum. We chose to include seven coefficients in our expansion for $\delta$.

on the ${ }^{12} \mathrm{C}$ foil and are listed in Table 2.

To optimize the spectrometer momentum reconstruction, we included a software wire chamber rotation about the central ray axis to align the detectors with respect to the spectrometer fields. This eliminates mixing the dispersive and non-dispersive coordinates and prevents a dependence of $\delta$ on measured transverse focal plane coordinates. The chamber rotation can be easily determined using this method since kinematic broadening, which will also create a term linear in $\phi_{T}$, is unimportant for the ${ }^{12} \mathrm{C}$ peak.

Figure 6 shows the OOPS momentum distribution on the carbon filament target at $\delta=0 \%$. The full width at half maximum (FWHM) resolution is $\approx 0.45 \%$ for the carbon filament target and does not vary appreciably across the focal plane. With a $5.0 \mathrm{~mm}$ beam spot size, we measured a resolution of $0.75 \%$ on a $26 \mathrm{mg} / \mathrm{cm}^{2}{ }^{12} \mathrm{C}$ foil.

The resolution obtained on the carbon filament and foil are consistent with estimates which 


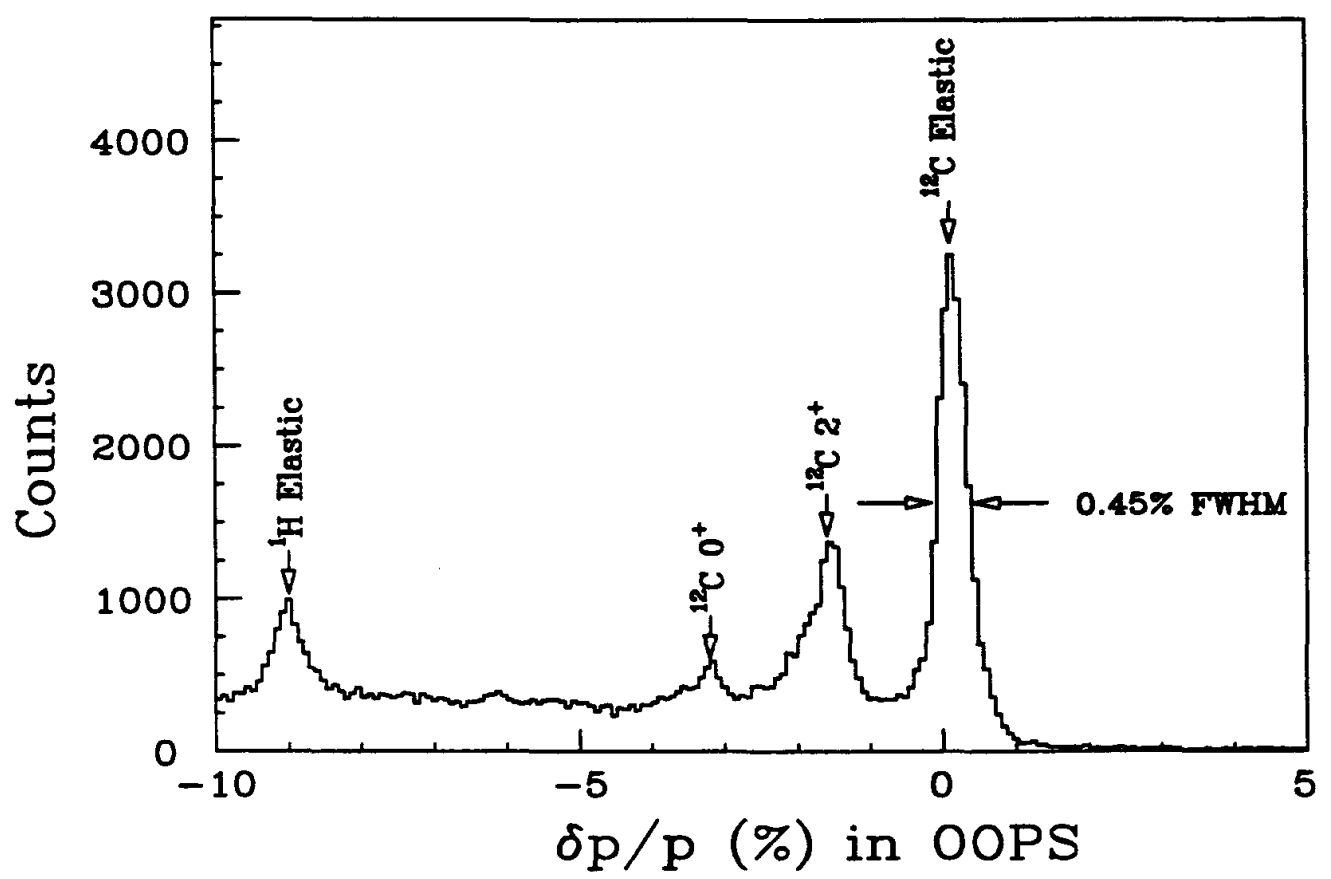

Figure 6: The momentum resolution for OOPS is shown on a carbon filament target of diameter $1.0 \mathrm{~mm}$.

fold in the target interaction size, the energy spread of the beam, multiple scattering in the target chamber and spectrometer vacuum windows, and the focal plane coordinate measurement uncertainties. In principle, momentum resolution better than $0.5 \%$ can be obtained by matching the dispersion of the beam on a target foil to the dispersion of the spectrometer. It is difficult in practice, however, to achieve the extremely small beam spot on the target which is needed to match the OOPS dispersion. Thus, the resolution of the OOPS will be governed by the interaction size on the target and the energy spread of the beam (see Table 1).

For the $300 \mathrm{MeV}$ electrons, the OOP spectrometer shows angular resolutions of better than $1.5 \mathrm{mr}$ reconstructed at the position of the sieve slit, which sits in the front collimator inside the spectrometer vacuum. This is in agreement with our projections for the intrinsic resolution of an OOPS coupled to the scattering chamber. In general, the spectrometer will not be coupled to the target vacuum chamber, and multiple scattering in the chamber exit 
window, air drift, and spectrometer entrance window will compete with the beam spot size on the target to drive the resolution in both the dispersive and non-dispersive angles.

Figure 7 shows a two-dimensional histogram of OOPS focal plane angles for electrons in the

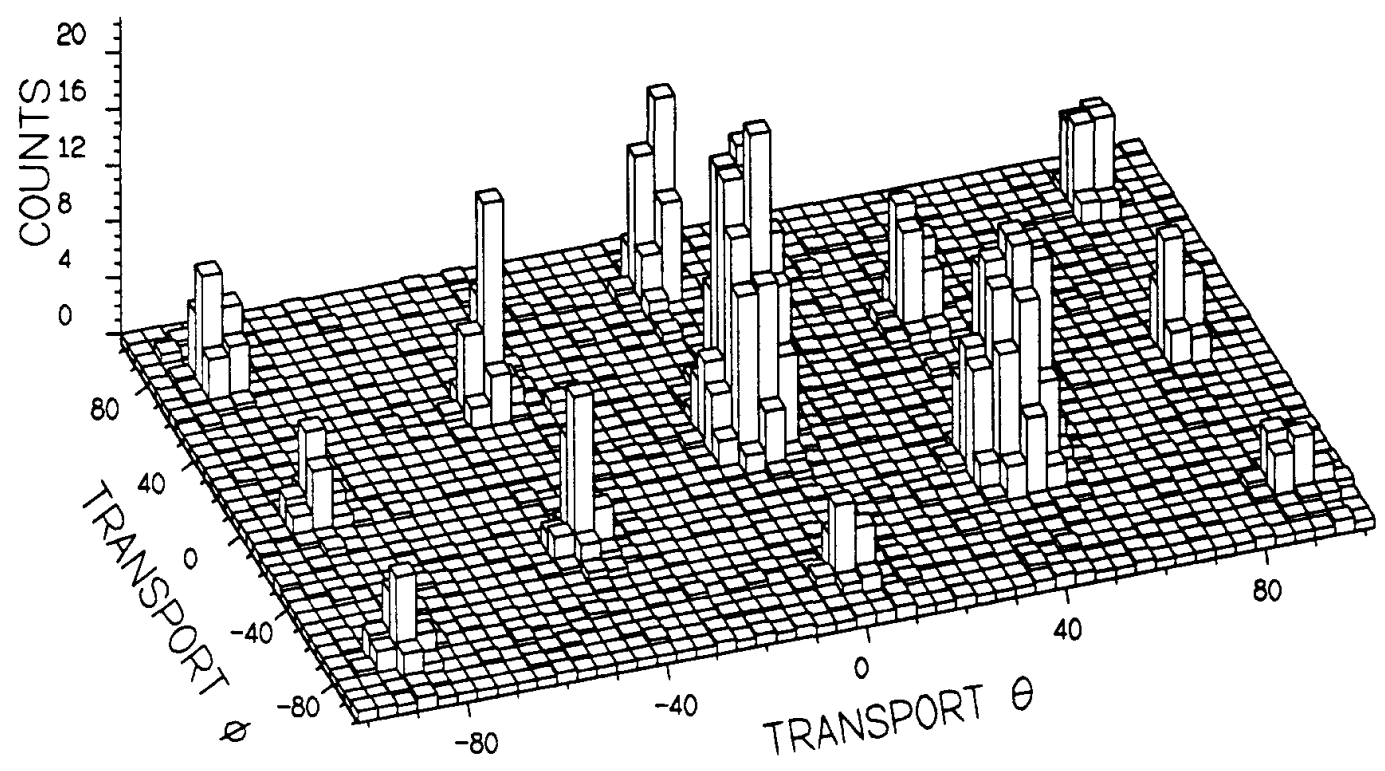

Figure 7: This two dimensional plot of OOPS focal plane angles for an elastic ${ }^{12} \mathrm{C}$ peak with a sieve slit installed in the spectrometer's front collimator shows that we could clearly identify the separate holes at the focal plane and thus reconstruct events to the target.

${ }^{12} \mathrm{C}$ elastic peak from the carbon filament target with a sieve slit installed in the front OOPS aperture. Since the holes are well separated in the sieve slit image, mapping the events back to the target is a straightforward task.

By creating a plot similar to Figure 5 for the dispersive angle, we chose to include three coefficients for the reconstruction of this variable. These coefficients agree very well with those derived from TRANSPORT and TURTLE and are listed in Table 2. The $1.5 \mathrm{mr}$ FWHM of a one-dimensional distribution of reconstructed $\theta_{T}$ angles for electrons passing through a single sieve slit hole is consistent with the angular size of the hole as viewed from the target, so the intrinsic spectrometer resolution in $\theta_{T}$ is at least this good. However, this 
is the resolution for a spectrometer which is coupled to the target chamber. In most cases, the resolution of the dispersive angle in OOPS will be dominated by the beam spot size and multiple scattering at the front of the spectrometer (see Table 1).

We found that a single matrix element rather nicely describes the non-dispersive angle. The FWHM of a $\phi_{T}$ distribution of events passing through a single sieve hole is again consistent with the angular size of the hole. The measured first order matrix elements for $\phi_{T}$ match those of the point target reverse matrix of Equation 3. By repeating the procedure on pseudo-data generated with our TURTLE model, we found that the solution converges on the extended target reverse matrix of Equation 2 in the absence of multiple scattering and measurement error. This solution is not very robust, however. The error in the extended target solution is driven by the OOPS detector angular measurement uncertainty (including multiple scattering):

$$
\begin{aligned}
\delta \phi_{T}^{2} & =16.21^{2} \delta y_{F}^{2}+2.289^{2} \delta \phi_{F}^{2} \\
& \approx 2.289^{2} \delta \phi_{F}^{2}
\end{aligned}
$$

The point target solution is driven by the length of the target interaction region in the non-dispersive plane:

$$
\begin{aligned}
\delta \phi_{T}^{2} & =1.103^{2} \delta y_{F}^{2}+2.524^{2} \delta y_{T}^{2} \\
& \approx 2.524^{2} \delta y_{T}^{2}
\end{aligned}
$$

Thus, the point target solution will have a smaller error as long as $\delta y_{T}(\mathrm{~cm})<\delta \phi_{F}(m r)$. For all but very extended targets (e.g., internal targets), the point target solution for $\phi_{T}$ is superior to the normal TRANSPORT reverse matrix solution.

As in the case of $\theta_{T}$, the resolution in $\phi_{T}$ will be driven by the beam spot size and the multiple scattering at the front of the spectrometer (see Table 1).

The data we have taken does not include the information needed to attempt a reconstruction of the variable $y_{T}$. Our expected resolution in this variable, however, is very poor - a first 
order error analysis suggests a resolution of about $3.3 \mathrm{~cm}$. Moreover, TURTLE simulations show that it would be very difficult to obtain the matrix elements for this variable with such poor resolution.

In a typical experimental geometry, the OOP spectrometers will be arranged symmetrically about the momentum transfer vector and will not all view the target in the same way. For a spectrometer on the beam line side of the momentum transfer vector, the angle with respect to the beam line can be very small. The effective target length, which is the projection of the actual target length onto a plane which is transverse to the spectrometer central ray, becomes very short for small spectrometer angles. This makes it difficult to depend on the proton spectrometers to determine the interaction point in the target. It is better to use the electron spectrometer for this purpose. The OHIPS spectrometer, which will often be used in conjunction with the OOPS system in the South Experimental Hall at Bates Laboratory, has excellent transverse target position resolution with the proper focal plane instrumentation.

\section{Efficiency Studies}

This section describes the absolute spectrometer efficiency profiles as functions of momentum and the transverse target position. The momentum efficiency profile is well understood over a bite of about $20 \%$. The extended target efficiency agrees only qualitatively with our model calculations.

The OOPS module has a rather large momentum bite. Design calculations show a region of flat efficiency (>90\%) over a range of roughly $15 \%$ of the value of the central momentum. The momentum efficiency profile is dependent upon the vertical opening of the spectrometer front collimator. We designed the collimator in the prototype OOPS to maximize solid angle while still achieving $100 \%$ efficiency at the central momentum. A flatter efficiency profile can be obtained by reducing the spectrometer vertical acceptance [1]. 
We measured the efficiency of OOPS using the $\mathrm{H}\left(e, \mathrm{e}^{\prime} \mathrm{p}\right)$ reaction by 'tagging' the recoiling proton detected in OOPS with elastically scattered electrons detected in the ELSSY spectrometer. The remotely controlled ELSSY solid angle slits allowed us to manipulate the projection of coincident proton onto OOPS. By varying the OOPS field, we then mapped the efficiency as a function of $\delta p / p$.

The relative OOPS efficiency in this scheme can be written as

$$
\epsilon^{O O P S}=\frac{N\left[H\left(e, e^{\prime} p\right)\right]}{N\left[H\left(e, e^{\prime}\right)\right]} \cdot \frac{\operatorname{Live}\left[H\left(e, e^{\prime}\right)\right]}{\operatorname{Live}\left[H\left(e, e^{\prime} p\right)\right]}
$$

where the first term is the ratio of coincident counts to electron singles in the hydrogen peak from elastic scattering on $\mathrm{CH}_{2}$, and the second term is the ratio of live times measured by scalers during the experiment. We performed one measurement on $\mathrm{a}^{12} \mathrm{C}$ target to obtain the ratio of carbon counts in the hydrogen super-elastic region to those in the elastic window.

We found a rather large and unexpected inefficiency of about $13 \%$. More than half of this inefficiency was traced to the OOPS trigger electronics network. A combination of long logic pulse widths and high scintillator accidental rates caused a rate-dependent inefficiency. This effect is well understood and will not plague future measurements. Roughly one percent of the missing efficiency can be attributed to multiple scattering, which will only decrease the relative spectrometer efficiency for two-body kinematics. We attribute the remaining three to five percent to scintillator inefficiency, which is perhaps threshold and rate dependent.

Figure 8 compares the shape of the measured OOPS momentum efficiency profile with our model calculations. The measured points have been scaled to unity at the central field. The error band in the plot is a normal single arm TURTLE calculation obtained by sending a white momentum spectrum into the spectrometer and binning events which manage to hit the last scintillator.

From our relative efficiency studies using two-body kinematics, we conclude that we understand the shape of the momentum efficiency profile very well. The absolute normalization 


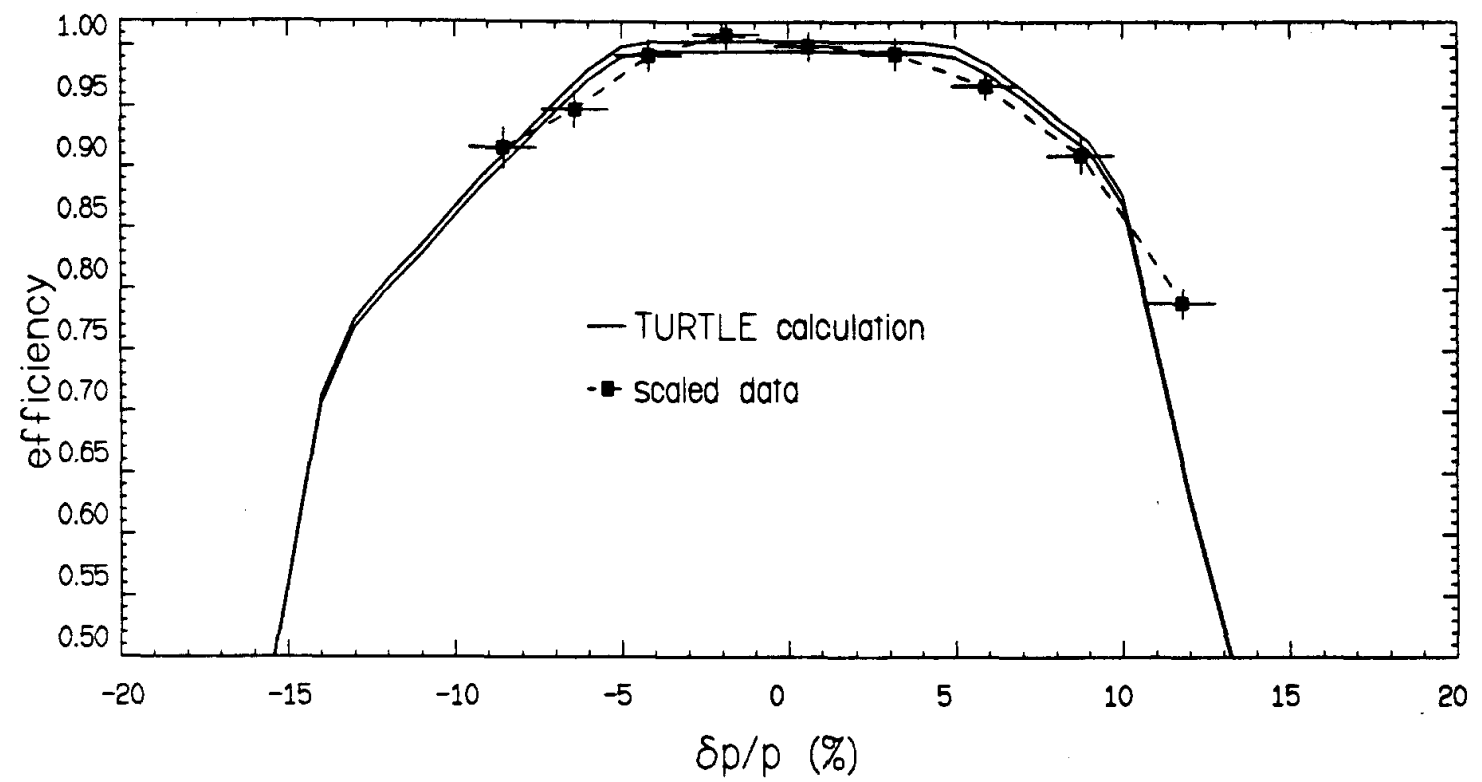

Figure 8: The points are measured efficiencies which are scaled to unity after the application of a calculated rate correction factor. The error band is the result of a TURTLE simulation.

of the spectrometer is understood at the level of a few percent, and future measurements should improve upon this.

Measurements with the OOPS will often use liquid targets with an extended geometry along the beam, so it is important to understand the efficiency versus this degree of freedom. The collimator which was installed in the prototype OOPS maximizes the transverse angular acceptance for a point target. The resulting extended target efficiency profile has no flat region. However, by decreasing the front collimator horizontal aperture, the efficiency profile can be flattened in the center at the expense of solid angle. This is discussed in the OOPS design report [1]. Preliminary measurements of the extended target efficiency using a $\mathrm{BeO}$ slant target show a qualitative agreement with TURTLE model calculations, but more precise tests are required to further define this profile prior to measurements which employ extended targets in absolute measurements or in relative measurements involving multiple OOPS. 


\section{Conclusions}

We have designed a four spectrometer system optimized for out-of-plane measurements. A prototype spectrometer module has been constructed, and its measured performance closely matches the design parameters. A momentum resolution better than $0.5 \%$ has been obtained, consistent with our design goals and a first order analysis which includes all measurement errors. The angular resolution of the spectrometer is driven by multiple scattering in the target chamber and front spectrometer vacuum windows; in the absence of these windows, calculations show that target coordinate angular resolutions as good as $1 \mathrm{mr}$ are possible. The spectrometer momentum efficiency profile matches the calculated shape over a momentum bite of about $20 \%$. The extended target efficiency profile agrees qualitatively with the expected shape.

\section{Acknowledgements}

This work was supported in part by the National Science Foundation under grant PHY 89-21146 and by the Department of Energy under grant DE-AC02-76ER03069.

\section{References}

[1] S. M. Dolfini et. al., to be submitted to Nuclear Instruments and Methods, 1993.

[2] J. E. Spencer and H. A. Enge, Nuclear Instruments and Methods 49, 181 (1967).

[3] H. A. Enge and S. B. Kowalski, $3^{\text {rd }}$ International Conference on Magnet Technology, 1970, p. 103.

[4] D. Jordan et al., Internal report, Bates Linear Accelerator Center (unpublished). 
[5] L. G. Atencio, J. F. Amann, R. L. Boudrie, and C. L. Morris, Nuclear Instruments and Methods 187, 381 (1981).

[6] A. H. Walenta, Nuclear Instruments and Methods 151, 461 (1978).

[7] E. A. J. M. Offermann, C. D. Jager, and H. D. Vries, Nuclear Instruments and Methods A262, 298 (1987).

[8] D. C. Carey, TURTLE (Trace Unlimited Rays Through Lumped Elements): A Computer Program For Simulating Charged Particle Beam Transport Systems, Fermilab National Accelerator Laboratory, 1978, NAL-64. 
